$B B$

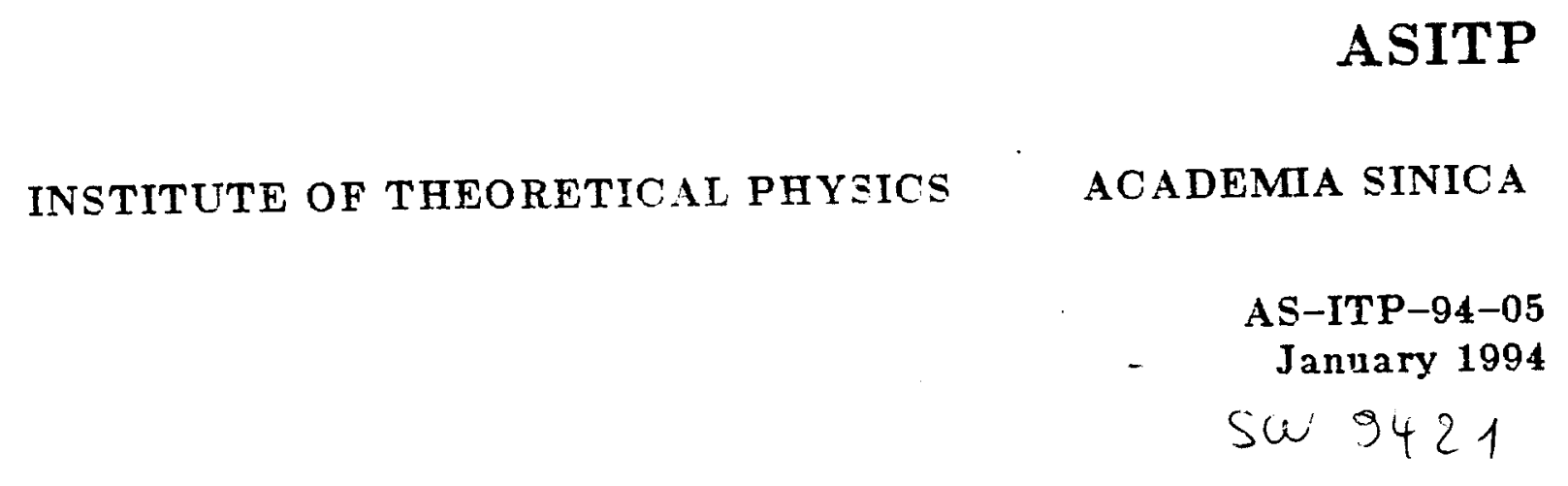

QCD INTERACTION IN PION-NUCLEUS

DCX REACTION

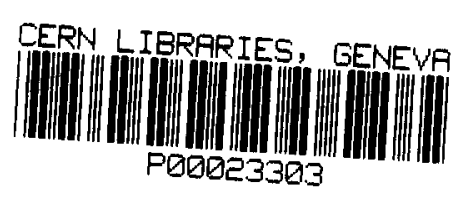

Xiao-fu LU En-guang ZHAO

Yu-xin LIU Jie MENG 


\section{QCD Interaction in Pion-Nucleus DCX Reaction}

Xiao-fu $L^{a, b, c}$, En-guang ZHAO ${ }^{a, c}$, Yu-xin LIU ${ }^{a, c}$ and Jie MENG ${ }^{a, c}$

a) CCAST(World Lab.), P. O. Box 8730, Beijing 100080, China

b) Department of Physics, Sichuan University, Chengdu 610064, China

c) Institute of Theoretical Physics, Academia Sinica, P. O. Box 2735 Beijing 100080, China ${ }^{3}$

\section{Abstract}

The Pion-nucleus double charge exchange(DCX) transition matrix element is under consideration in the framework of QCD. The interactions including the perturbative effect and non-perturbative contributions of gluon condensate and quark condensate in the DCX process are discussed. The interaction operators are given.

It canne as a big surprise that the first Pion-nucleus double charge exchange reaction (DCX) measurement below the $\Delta$-resonance was conducted in 1984. Navon et al ${ }^{[1]}$ and later Leitch et al ${ }^{[2]}$ measured the DIAS transition on ${ }^{14} \mathrm{C}$ at an incident pion energy of $\mathrm{T}_{\pi}=50 \mathrm{MeV}$ and found that the forward angle cross section is as large as at $T_{\pi}=300 \mathrm{MeV}$ and even is close to the forward angle cross section for SCX on ${ }^{14} \mathrm{C}$ at $T_{\pi}=50 \mathrm{MeV}$. This observation was in clear contradiction to expectations. Since the destructive interference of $s$ - and p- waves in the $\pi N$ - system causes the SCX cross section to undergo a deep minimum near $T_{\pi}=50 \mathrm{MeV}$, one could speculate that something similar would also happen for DCX processes. In order to account for this unexpectedly large DIAS cross section on ${ }^{14} \mathrm{C}$ at $T_{\pi}=50 \mathrm{MeV}$, non-nucleonic processes, such as the double charge exchange on six quark clusters ${ }^{[3]}$, were proposed at first. However. conventional explanations $s^{[4]}$ have also been found soon, based on a careful treatment of nucleon-nucleon correlations. Nevertheless, a common feature of both exotic and conventional explanations is that both these treatments stress the relevance of short distances $(\leq 1 \mathrm{fm})$ between the nucleons participating in the DCX process ${ }^{[s]}$. This is a region in which nucleons overlap strongly so that the conventional multiple scattering treatment may be an oversimplification. A more correct consideration of the physics occurring at such short distance from quark degrees of freedom is then necded.
The main purpose of this paper is to develop a more sophisticated QCD treatment for the two nucleon DCX transition matrix elements, especially to take into account some non-perturbative effects

The S-matrix of DCX reaction in the Heisenberg Representation can be expressed as

$$
\left\langle p p \pi^{-}|S| n n \pi^{+}\right\rangle=\frac{1}{(2 \pi)^{3} 2 \sqrt{\omega_{\mathbf{k}} \omega_{\mathbf{k}^{\prime}}}} \int d^{4} x \int d^{4} x^{\prime} e^{i\left(\mathbf{k}^{\prime} \cdot \mathbf{x}^{\prime}-\mathbf{k} \cdot \mathbf{x}\right)}
$$

$$
\left(\square_{x}+m_{\pi}^{2}\right)\left(\square_{x^{\prime}}+m_{\pi}^{2}\right)\left\langle p p\left|T\left(\phi_{\pi^{-}}\left(x^{\prime}\right) \phi_{\pi^{-}}(x)\right)\right| n n\right\rangle,
$$

where the inatrix elements can be expressed in figure 1 , in which the dashed external lines represent the Green function for the $\phi_{\pi}-(x)$ field. Considering the Goldstone effect, we have

$$
\begin{aligned}
& \partial_{\mu} A_{u d}^{\mu}=i 2 m_{q} \bar{u}(x) \gamma_{5} d(x), \\
& \partial_{\mu} A_{u d}^{\mu}=\sqrt{2} f_{\pi} m_{\pi}^{2} \phi_{\pi}-(x),
\end{aligned}
$$

where $A_{u d}^{\mu}=\bar{u} \gamma^{\mu} \gamma_{5} d$, From the dynamical spontaneous breaking of chiral symmetry we have the effective Lagrangian term ${ }^{[0]}$

$$
-i \frac{\sqrt{2} m_{q}}{f_{\pi}} \bar{u} \gamma_{5} d \phi_{\pi}+(x)
$$

Using the equation

$$
\left(\square_{x}+m_{\pi}^{2}\right) \triangle_{F}=-i \delta(x-y),
$$

where $\triangle_{F}$ is the Feynman propagator for pions, we can get

$$
\left\langle p p \pi^{-}|S| n n \pi^{+}\right\rangle=\frac{-2}{f_{\pi}^{2}} \frac{1}{2(2 \pi)^{3} \sqrt{\omega_{\mathbf{k}} \omega_{\mathrm{k}^{\prime}}}} \int d^{4} x \int d^{4} x^{\prime} e^{i\left(\mathrm{k}^{4} \cdot \mathbf{x}^{\prime}-\mathrm{k} \cdot \mathbf{x}\right)}
$$$$
\left\langle p p\left|T\left(m_{q} \bar{u} \gamma_{s} d\left(x^{\prime}\right) m_{q} \bar{u} \gamma_{s} d(x)\right)\right| n n\right\rangle .
$$

Making a translation transformation we have

$$
\left\langle p p \pi^{-}|S| n n \pi^{+}\right\rangle=\frac{-2}{f_{\pi}^{2}} \frac{2 \pi}{2 \sqrt{\omega_{\mathbf{k}} \omega_{\mathbf{k}^{\prime}}}} \delta\left(k^{\prime}+p^{\prime}-k-p\right) \int d^{4} x e^{i\left(\mathbf{k}^{\prime} \cdot \mathbf{x}\right)}
$$$$
\left\langle p p\left|T\left(m_{q} \bar{u} \gamma_{5} d(x), m_{7} \bar{u} \gamma_{5} d(0)\right)\right| n n\right\rangle,
$$

where $k^{\prime}$ is the ontgoing momentum of $\pi^{-}, k$ is the incident momentum, $p^{\prime}$ is the momentum of the nucleons ( $p \mathrm{p}), p^{\prime}$ is the momentum of the nucleons ( $\mathrm{nn}$ ). 
We emphasize that the above discussion is based on the theory of chiral symmetry breaking. In order to carry out the calculation we shall use the QCD sum rule. According to the operator product expansion, we have

$$
\begin{aligned}
& T\left(\bar{u}(x) \gamma_{s} u(x) \bar{u}(0) \gamma_{s} d(0)\right) \\
= & : \bar{u}(x) \gamma_{s} d(x) \bar{u}(0) \gamma_{s} d(0): \\
& +: \bar{u}(0) \gamma_{s} d(0) \bar{u}(0) \gamma_{s} d(0): C_{G}(x): G_{\mu \nu}^{a} G_{\mu \nu}^{a}: \\
& +: \bar{u}(0) \gamma_{s} d(0) \bar{u}(0) \gamma_{5} d(0): C_{Q}(x) m_{q}: \bar{q}(0) q(0): \\
& +\cdots \cdots
\end{aligned}
$$

The first term in the right hand side is the perturbative effect, which has the contribution

$$
m_{q}^{2} \int d^{4} x e^{i\left(\mathbf{k}^{\prime} \cdot \mathbf{x}\right)}\left\langle p p\left|: \bar{u} \gamma_{5} d(x), \bar{u} \gamma_{5} d(0):\right| n n\right\rangle .
$$

Because of the energy momentum conservation the low order contributions of eq.(7) can be represented in figure 2 .

It is obvious that the contributions of Fig.2(a1-a4) are $\frac{a^{2}}{a^{2}}$, and those from Fig.2(bl-b4) and Fig.2.(c1-c4) are $\frac{q^{4}}{a^{4}}$. As only the lowest order contributions of the perturbative effect are taken into account, figures 2(b1-b4) and (cl-c4) can be ignored. However, the loops in figures 2(b1-b4) and (c1-c4) can be broken, and the broken lines can be condensed. The non-perturbative effects of gluon condensate and quark condensate then come naturally. The corresponding interactions are shown in figure 3 and figure 4 respectively.

For Fig.2(al), the contribution is

$$
\begin{gathered}
S_{(2, a 1)}^{(2)}=-i \bar{u}\left(p_{1}^{\prime}\right) g \frac{\lambda^{a}}{2} \gamma_{\mu} \frac{p_{1}-k^{\prime}+m}{\left(p_{1}-k^{\prime}\right)^{2}-m^{2}} \gamma_{5} \frac{1}{\left(p_{1}-k^{\prime}-p_{1}^{\prime}\right)^{2}} d\left(p_{1}\right) \\
\bar{u}\left(p_{2}^{\prime}\right) g \frac{\lambda^{\mathbf{a}}}{2} \gamma^{\mu} \frac{p_{2}+\not k+m}{\left(p_{2}+k\right)^{2}-m^{2}} \gamma_{5} d\left(p_{2}\right) .
\end{gathered}
$$

For Fig.2.(a2), the contribution is

$$
\begin{gathered}
S_{(\dot{2}, a 2)}^{(2)}=-i \bar{u}\left(p_{1}^{\prime}\right) \gamma_{5} \frac{p_{1}^{\prime}+k^{\prime}+m}{\left(p_{1}^{\prime}+k^{\prime}\right)^{2}-m^{2}} g \frac{\lambda^{a}}{2} \gamma_{\mu} \frac{1}{\left(p_{1}-k^{\prime}-p_{1}^{\prime}\right)^{2}} d\left(p_{1}\right) \\
\bar{u}\left(p_{2}^{\prime}\right) g \frac{\lambda^{a}}{2} \gamma^{\mu} \frac{p_{2}+k+m}{\left(p_{2}+k\right)^{2}-m^{2}} \gamma_{5} d\left(p_{2}\right) .
\end{gathered}
$$

For Fig.2.(a3), the contribution is

$$
\begin{gathered}
S_{(2, a 3)}^{(2)}=-i \bar{u}\left(p_{1}^{\prime}\right) g \frac{\lambda^{a}}{2} \gamma_{\mu} \frac{1}{\left(p_{1}-k^{\prime}-p_{1}^{\prime}\right)^{2}} \frac{p_{1}-\mu^{\prime}+m}{\left(p_{1}-k^{\prime}\right)^{2}-m^{2}} \gamma_{5} d\left(p_{1}\right) \\
\\
\bar{u}\left(p_{2}^{\prime}\right) \gamma_{5} \frac{\left(p_{2}^{\prime}-k+m\right.}{\left(p_{2}^{\prime}-k\right)^{2}-m^{2}} g \frac{\lambda^{a}}{2} \gamma^{\mu} d\left(p_{2}\right) .
\end{gathered}
$$

For Fig.2.(a4), the contribution is

$$
\begin{gathered}
S_{(2, a+1)}^{(2)}=-i \bar{u}\left(p_{1}^{\prime}\right) \gamma_{5} \frac{\not p_{1}^{\prime}+k^{\prime}+m}{\left(p_{2}^{\prime}+k^{\prime}\right)^{2}-m^{2}} g \frac{\lambda^{a}}{2} \gamma_{\mu} \frac{1}{\left(p_{1}-k^{\prime}-p_{1}^{\prime}\right)^{2}} d\left(p_{1}\right) \\
\bar{u}\left(p_{2}^{\prime}\right) \gamma_{5} \frac{p_{2}^{\prime \prime}-k+m}{\left(p_{2}^{\prime}-k\right)^{2}-m^{2}} g \frac{\lambda^{a}}{2} \gamma^{\mu} d\left(p_{2}\right) .
\end{gathered}
$$

For calculating the second term of the time-ordered product expansion, namely the contribution of gluon condensate, we adopt the fixed-point gauge technique $e^{[7]}$. In this method we introduce an external gauge field $A_{\mu}^{a}(x)$ with the gauge condition $x^{\mu} A_{\mu}^{a}(x)=0$. The external gauge field $A_{\mu}^{a}(x)$ can then be expressed directly in terms of gauge covariant quantities

$$
A_{\mu}(x)=\frac{1}{2} x^{\nu} G_{\nu \mu}(0)+\frac{1}{3} x^{\alpha} x^{\nu} D_{\alpha} G_{\nu \mu}(0)+\cdots
$$

To calculate the contribution of gluon condensate we shall employ the diagrams shown in figure 3 and figure 5. From the figures we know that the contributions of figure 3 are of the order of $\left\langle G_{\mu \nu}^{a} G_{\mu \nu}^{a}\right\rangle \frac{g^{4}}{q^{4}} \sim \alpha, \frac{\left\langle\alpha_{0} G_{\mu \nu}^{a} G_{\mu \nu}^{a}\right\rangle}{q^{4}}$. The contributions of figure 5 are of the order of $\left\langle G_{\mu \nu}^{a} G_{\mu \nu}^{a}\right\rangle \frac{g^{8}}{q^{4}} \sim \alpha_{3}^{2} \frac{\left\langle\alpha, G_{\mu \nu}^{a} G_{\mu \nu}^{a}\right\rangle}{q^{4}}$. Then in the first order approximation figure 5 can be neglected. The contribution of figure 3 can be expressed as

For Fig.3.(1),

$$
\begin{aligned}
S_{(3,1)}^{(2)}= & \bar{u}\left(p_{1}^{\prime}\right) g \frac{i \lambda^{a}}{2} \gamma_{\mu} \frac{-i g^{\mu \delta}}{q^{2}}\left[\frac{9}{64} \frac{-i g^{2}}{q^{2}} \delta^{a b} g_{\delta \lambda}\left\langle G_{\mu \nu}^{a} G_{\mu \nu}^{a}\right)\right] \frac{i}{p_{1}-k^{\prime}-m} \gamma_{5} d\left(p_{1}\right) \\
& \frac{-i g^{\lambda \nu}}{q^{2}} \bar{u}\left(p_{2}^{\prime}\right) g \frac{i \lambda^{b}}{2} \gamma_{\nu} \frac{i}{p_{2}+k-m} \gamma_{5} d\left(p_{2}\right) \\
= & \frac{9 i}{64} \frac{g^{4}}{q^{4}} \frac{\lambda^{a}}{2} \frac{\lambda^{a}}{2}\left\langle G_{\mu \nu}^{a} G_{\mu \nu}^{a}\right\rangle \\
& {\left[\bar{u}\left(p_{1}^{\prime}\right) \gamma_{\mu} \frac{p_{1}-k^{\prime}+m}{\left(p_{1}-k\right)^{2}-m^{2}} \gamma_{5} d\left(p_{1}\right) \frac{1}{q^{2}} \bar{u}\left(p_{2}^{\prime}\right) \gamma^{\mu} \frac{p_{2}+k+m}{\left(p_{2}+k\right)^{2}-m^{2}} \gamma_{5} d\left(p_{2}\right)\right] }
\end{aligned}
$$

For Fig.3.(2),

$$
\begin{aligned}
S_{(3,2)}^{(2)}= & \frac{9 i}{64} \frac{g^{4}}{q^{4}} \frac{\lambda^{a}}{2} \frac{\lambda^{a}}{2}\left\langle G_{\mu \nu}^{a} G_{\mu \nu}^{a}\right\rangle \\
& {\left[\bar{u}\left(p_{1}^{\prime}\right) \gamma_{s} \frac{p_{1}^{\prime}+k^{\prime}+m}{\left(p_{1}^{\prime}+k^{\prime}\right)^{2}-m^{2}} \gamma_{\mu} d\left(p_{1}\right) \frac{1}{q^{2}} \bar{u}\left(p_{2}^{\prime}\right) \gamma^{\mu} \frac{p_{2}+k+m}{\left(p_{2}+k\right)^{2}-m^{2}} \gamma_{5} d\left(p_{2}\right)\right] . }
\end{aligned}
$$


For Fig.3.(3),

$$
\begin{aligned}
S_{(3,3)}^{(2)}= & \frac{9 i}{64} \frac{g^{4}}{q^{4}} \frac{\lambda^{a}}{2} \frac{\lambda^{a}}{2}\left\langle G_{\mu \nu}^{a} G_{\mu \nu}^{a}\right\rangle \\
& {\left[\bar{u}\left(p_{1}^{\prime}\right) \gamma_{\mu} \frac{1}{q^{2}} \frac{p_{1}^{\prime}-k^{\prime}+m}{\left(p_{1}^{\prime}-k^{\prime}\right)^{2}-m^{2}} \gamma_{5} d\left(p_{1}\right) \bar{u}\left(p_{2}^{\prime}\right) \gamma_{5} \frac{p_{2}^{\prime}-k+m}{\left(p_{2}^{\prime}-k\right)^{2}-m^{2}} \gamma^{\mu} d\left(p_{2}\right)\right] . }
\end{aligned}
$$

For Fig.3.(4),

$$
\begin{aligned}
S_{(3,4)}^{(2)}= & \frac{9 i}{64} \frac{g^{4}}{q^{4}} \frac{\lambda^{a}}{2} \frac{\lambda^{a}}{2}\left\langle G_{\mu \nu}^{a} G_{\mu \nu}^{a}\right\rangle \\
& {\left[\bar{u}\left(p_{1}^{\prime}\right) \gamma_{5} \frac{1}{q^{2}} \frac{\not p_{2}^{\prime}+k^{\prime}+m}{\left(p_{1}^{\prime}+k^{\prime}\right)^{2}-m^{2}} \gamma_{\mu} d\left(p_{1}\right) \bar{u}\left(p_{2}^{\prime}\right) \gamma_{5} \frac{p_{2}^{\prime}-k+m}{\left(p_{2}^{\prime}-k\right)^{2}-m^{2}} \gamma^{\mu} d\left(p_{2}\right)\right] }
\end{aligned}
$$

The third term of eq.(6) is the contribution of quark condensate. It can be shown that it is sufficient to consider the diagrams in figure 4 for calculating the lowest order of this contribution.

The contribution from Fig.4(1) can be written in the following formula,

$$
\begin{aligned}
S_{(4,1)}^{(2)}= & \bar{u}\left(p_{1}^{\prime}\right) g \frac{i \lambda^{a}}{2} \gamma_{\mu} \frac{-i g^{\mu \delta}}{q^{2}}\left[\frac{1}{6}\langle\psi \psi\rangle \frac{i m g^{2}}{q^{2}} \delta_{a b}\left(\frac{q^{\delta} q^{\lambda}}{q^{2}}-g^{\delta \lambda}\right)\right] \frac{i}{p_{1}-k^{\prime}-m} \gamma_{5} d\left(p_{1}\right) \\
& \frac{-i g^{\lambda \nu}}{q^{2}} \bar{u}\left(p_{2}^{\prime}\right) g \frac{i \lambda^{b}}{2} \gamma_{\nu} \frac{i}{p_{2}+k-m} \gamma_{5} d\left(p_{2}\right) \\
= & \frac{1}{6} \frac{i}{q^{4}}\langle m \psi \psi\rangle g^{\prime} \frac{\lambda^{a}}{2} \frac{\lambda^{a}}{2}\left[\bar{u}\left(p_{1}^{\prime}\right) \gamma_{\mu} \frac{1}{q^{2}} \frac{p_{1}-k^{\prime}+m}{\left(p_{1}-k^{\prime}\right)^{2}-m^{2}} \gamma_{5} d\left(p_{1}\right)\right. \\
& \left.\left(g^{\mu \nu}-\frac{q^{\mu} q^{\nu}}{q^{2}}\right) \bar{u}\left(p_{2}^{\prime}\right) \gamma_{\nu} \frac{p_{2}+k+m}{\left(p_{2}+k\right)^{2}-m^{2}} \gamma_{5} d\left(p_{2}\right)\right] .
\end{aligned}
$$

For Fig.4.(2), (3) and (4), we have

$$
\begin{aligned}
& S_{(4,2)}^{(2)}=\frac{1}{6} \frac{i}{q^{4}}\langle m \psi \psi\rangle g^{4} \frac{\lambda^{a}}{2} \frac{\lambda^{a}}{2}\left[\bar{u}\left(p_{1}^{\prime}\right) \gamma_{5} \frac{1}{q^{2}} \frac{p_{1}^{\prime}+k^{\prime}+m}{\left(p_{1}^{\prime}+k^{\prime}\right)^{2}-m^{2}} \gamma_{\mu} d\left(p_{1}\right)\right. \\
& \left.\left(g^{\mu \nu}-\frac{q^{\mu} q^{\nu}}{q^{2}}\right) \vec{u}\left(p_{2}^{\prime}\right) \gamma_{\nu} \frac{p_{2}+k+m}{\left(p_{2}+k\right)^{2}-m^{2}} \gamma_{5} d\left(p_{2}\right)\right] \text {, } \\
& S_{(4,3)}^{(2)}=\frac{1}{6} \frac{i m}{q^{4}}\left\langle m \psi^{\prime} \psi\right\rangle g^{4} \frac{\lambda^{a}}{2} \frac{\lambda^{a}}{2}\left[\bar{u}\left(p_{1}^{\prime}\right) \gamma_{\mu} \frac{1}{q^{2}} \frac{\not p_{1}^{\prime}-k^{\prime}+m}{\left(p_{1}^{\prime}-k^{\prime}\right)^{2}-m^{2}} \gamma_{5} d\left(p_{1}\right)\right. \\
& \left.\left(g^{\mu \nu}-\frac{q^{\mu} q^{\nu}}{q^{2}}\right) \bar{u}\left(p_{2}^{\prime}\right) \gamma_{5} \frac{p_{2}^{\prime}-k+m}{\left(p_{2}^{\prime}-k\right)^{2}-m^{2}} \gamma_{\nu} d\left(p_{2}\right)\right] \text {, }
\end{aligned}
$$

$$
\begin{aligned}
S_{(4,4)}^{(2)}= & \frac{1}{6} \frac{i}{q^{4}}\langle m \psi \psi\rangle g^{4} \frac{\lambda^{a}}{2} \frac{\lambda^{a}}{2}\left[\bar{u}\left(p_{1}^{\prime}\right) \gamma_{5} \frac{1}{q^{2}} \frac{p_{1}^{\prime}+k^{\prime}+m}{\left(p_{1}^{\prime}+k^{\prime}\right)^{2}-m^{2}} \gamma_{\mu} d\left(p_{1}\right)\right. \\
& \left.\left(g^{\mu \nu}-\frac{q^{\mu} q^{\nu}}{q^{2}}\right) \bar{u}\left(p_{2}^{\prime}\right) \gamma_{5} \frac{p_{2}^{\prime}-k+m}{\left(p_{2}^{\prime}-k\right)^{2}-m^{2}} \gamma_{\nu} d\left(p_{2}\right)\right] .
\end{aligned}
$$

The expressions (8-11) and (13-20) show that, if the order of magnitude of the contribution of the perturbative effect is taken as 1 , the contribution of the gluon condensate and the quark condensate are respectively $M O_{g} \sim g^{4} \frac{\left(G^{a} G^{a}\right\rangle}{q^{4}}$ $\sim \alpha, \frac{\left\langle\alpha, G_{\mu \nu}^{a} G_{\mu \nu}^{a}\right\rangle}{q^{4}}, M O_{q} \sim g^{4} \frac{\langle m \psi \psi\rangle}{q^{4}} \sim \alpha_{s}^{2} \frac{\langle m \psi \psi\rangle}{q^{4}}$. Considering the reaction with incident energy $50 \mathrm{MeV}, M O_{g} \sim \alpha,\left(\frac{360}{50}\right)^{4} \sim 10^{-1} \times 10^{3} \sim 10^{2}, M O_{q} \sim \alpha_{3}^{2}\left(\frac{240}{50}\right)^{4}$ $\sim 10^{-2} \times 10^{2} \sim 1$. This result indicates that the non-perturbative contribution of the gluon condensate is very important in the Pion-nucleus DCX reaction, and that of quark condensate holds the same importance as the perturbative one. With the six quark wave functions of the two nucleons $(\mathrm{nn})$ and $(\mathrm{pp})$ in the nucleus being taken into account, the S-matrix element can be calculated explicitly. This work is in process. The numerical result will be given in the near future.

This work is supported by the National Natural Science Foundation of China and the grant LWTZ 1298 of the Chinese Academy of Sciences. One of the authors (Y.X.Liu) thanks also the support of the China Post-doctoral Science Foundation. The authors are grateful for the hospitality of CCAST and helpful discussions with Professors H. C. Chiang, F. Wang and C. H. Chang.

\section{REFERENCES}

[1] I. M. Navon, et. al., Phys. Rev. Lett. 52 (1984) 105.

[2] M. J. Leitch, et. al., Phys. Rev. Lett. 54 (1985) 1482.

[3] G. A. Miller, Phys. Rev. Lett. 53 (1984) 2008; ibid, Phys. Rev. C35 (1987) 377 .

[4] N. Auerbach, et. al., Phys. Rev. C38 (1988) 1277.

(5) H. Clement, Prog. Part. Nucl. Phys. 29 (1992) 175.

[6] R. T. Cahill and C. D. Roberts, Phys. Rev. D32 (1985) 2419

[7] L. J. Reinders, et. al., Phys. Rep. 127 (1985) 1. 


\section{Figure Captions:}

Fig. 1. The sketch of the interactions in DCX process.

Fig. 2. The perturbative interactions with one-gluon exchange.

Fig. 3. The non-perturbative interactions of gluon condensate with one-gluon exchange.

Fig. 4. The non-perturbative interactions of quark condensate with one-gluon exchange.

Fig. 5. The loop correction of gluon condensate with one-gluon exchange.

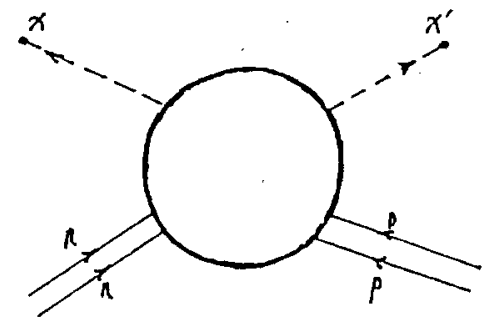

Fig.1.

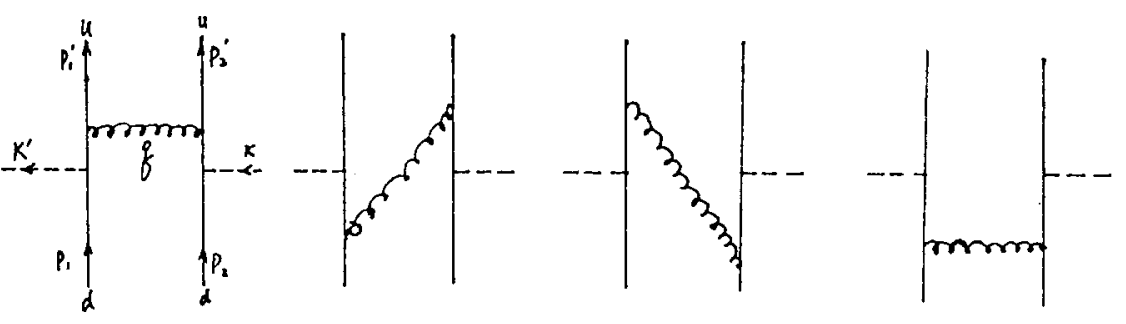

(ai)

(a2)

(a3)

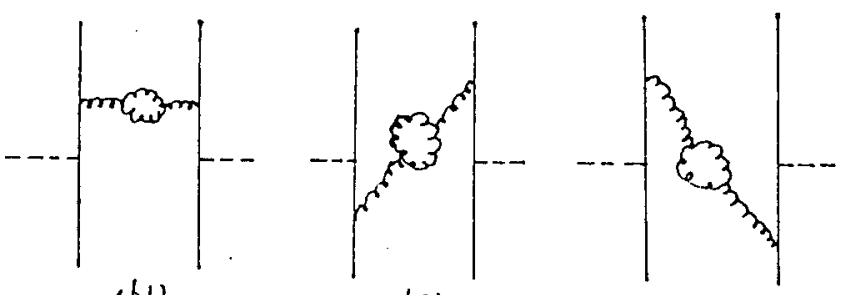

(b2)

(b3) (a4)

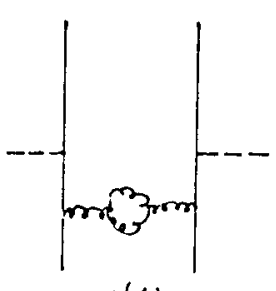

(b4)

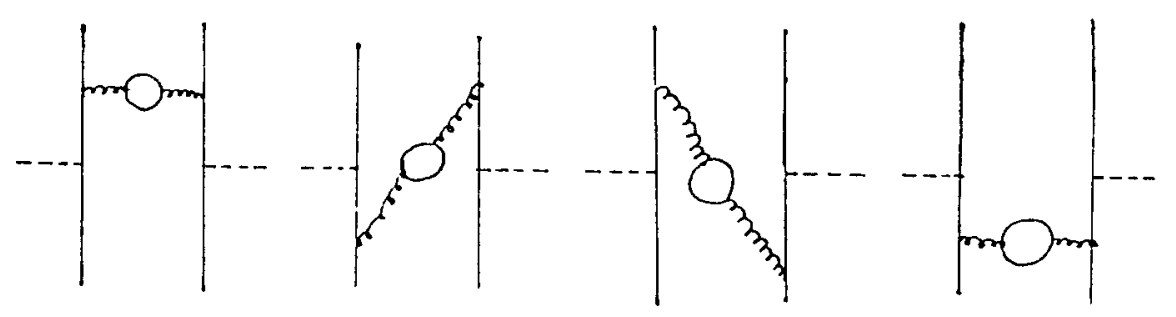

(c1)

(Cx)

(c)

(24)

Fig. 2. 


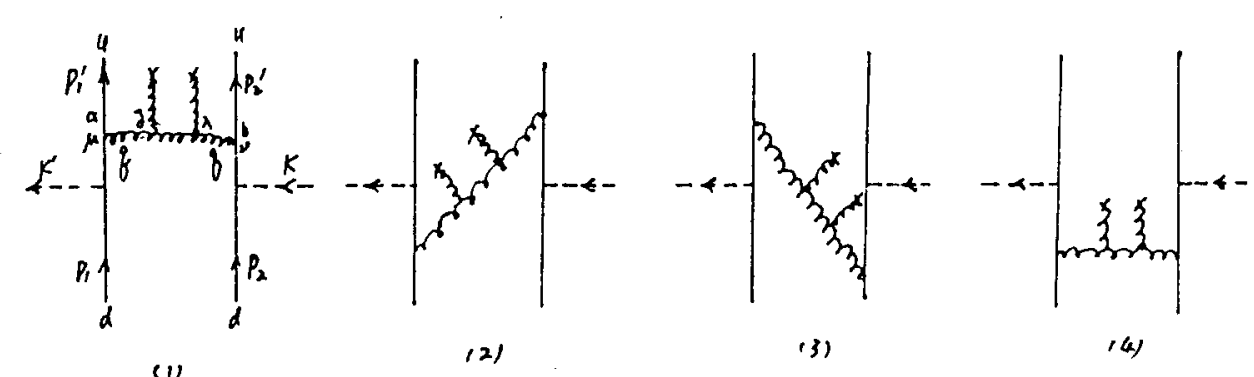

Fig. 3 :

$\left.\right|_{121} ^{a} p_{2}^{u} p_{21}^{n}$

Fig. 4.

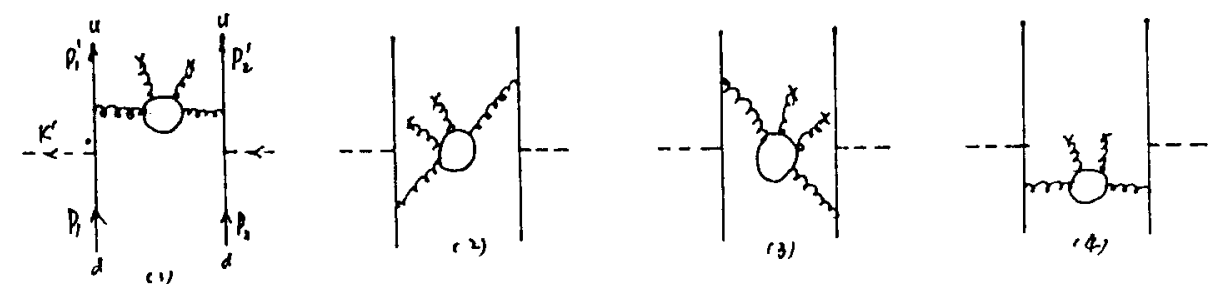

(6)

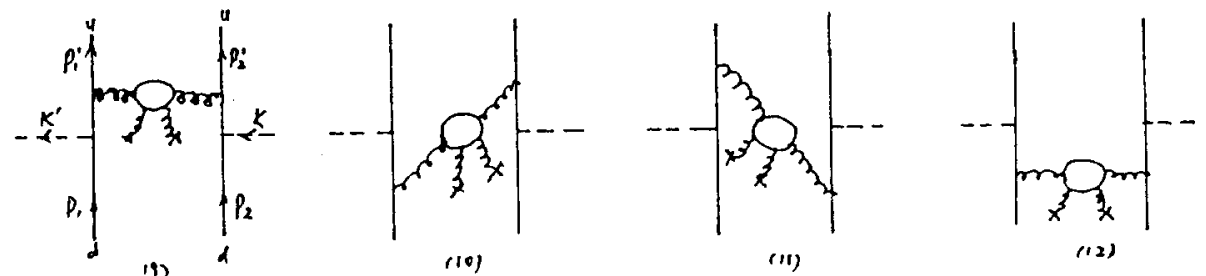

Fig. 5 